\title{
Zero-order suppression for two-photon holographic excitation
}

\section{Oscar Hernandez, ${ }^{1}$ Marc Guillon, ${ }^{1 *}$ Eirini Papagiakoumou, ${ }^{1,2}$ and Valentina}

\section{Emiliani $^{1}$}

\author{
${ }^{1}$ Wavefront-engineering Microscopy Group, Neurophotonics Laboratory CNRS \\ UMR8250, Paris Descartes University, Sorbonne Paris Cité, 45 rue des Saints-Pères, \\ 75270 Paris Cedex 06, France \\ ${ }^{2}$ Institut national de la santé et de la recherche médicale (Inserm) \\ *Corresponding author: marc.guillon@parisdescartes.fr
}

\begin{abstract}
Wavefront shaping with liquid crystal spatial light modulators (LC-SLMs) is frequently hindered by a remaining fraction of undiffracted light, the so-called "zero-order". This contribution is all the more detrimental in configurations for which the LC-SLM is Fourier conjugated to a sample by a lens, because in these cases this undiffracted light produces a diffraction-limited spot at the image focal plane. In this work we propose to minimize two-photon excitation of the sample resulting from this unmodulated light by introducing optical aberrations to the excitation beam. Aberrations are subsequently compensated by the LC-SLM but only for the modulated part of the beam, and not for the "zero-order" component. In order to experimentally demonstrate the method we use astigmatism as the optical aberration by simply adding one or two cylindrical lenses in the optical path of the beam. A $10^{4}$ decrease in zero-order-induced two-photon fluorescence intensity is demonstrated. Combining this approach with temporal focusing is shown to further decrease zero-order fluorescence by a factor of 100 .
\end{abstract}

230.6120, 090.1970, 070.4340.

Spatial Light Modulators (SLMs) are devices allowing the design of computer generated optical elements with high spatial resolution. Among these devices, phase-only modulators based on nematic liquid crystal matrices (LC-SLMs), modulate a large fraction of impinging energy and offer high diffraction efficiency, i.e. the ratio between the light power impinging on the LC-SLM, $P_{0}$, and the light diffracted into the shaped pattern $P_{\text {out }}$, because they can reproduce the calculated phase mask with sufficiently small quantization steps. They find applications in different research fields including optical trapping [1], photo-polymerization 
[2], lithography [3], holographic photostimulation of neuronal cells by photolysis[4] (add refs: Zahid et al. Plos One 2010, Yang et al. J. Neural Engineer. 2011) or optogenetics (add refs: Bègue et al. Biomed. Opt. Express 2013), adaptive optics[8], ultrashort pulse shaping [5], phase conjugation [6, 7], or focusing through turbid media [9].

Configurations in which the LC-SLM is conjugated to the back focal plane - or Fourier plane - of a lens $[1,2,3,4]$ permit an efficient re-distribution of impinging laser energy into arbitrarily shaped patterns. The phase profile addressed to the SLM is usually calculated using Fourier transform based iterative algorithms [11]. Existing LC-SLM devices permit nowadays to reach diffraction efficiency values, close to the limit imposed by theory, i.e. $\sim 95$ and $38 \%$ for $P_{\text {out }}$ directed at the center of the field of excitation or at the border, respectively. The remaining light is distributed among the higher diffracted orders and an undiffracted component. This undiffracted component, so-called "zero order", results in a tightly focused spot at the center of the field of excitation, the intensity of which, depending on the applied phase profile, can reach $25 \%$ of that of the incoming light [10]. This value can be reduced down to $2-5 \%$, regardless of the projected hologram, by performing a preliminary characterization of the LC-SLM [10]. Although only a small fraction of the total available power remains in the zero-order diffraction-limited spot, its contribution is all the more important in applications involving non-linear photo-physical processes, like two-photon excitation, due to the quadratic dependence of two-photon processes on the excitation intensity.

In the past, it has been proposed to remove the zero-order contribution by deviating the modulated component, $P_{\text {out }}$, away from the zero-order spot. Precisely, it has been shown that a lateral shift of the pattern of interest permits to remove the zero order contribution at the focal plane by placing at a sample conjugated plane a beam block centered on the zero-order position. This approach leaves however, an inaccessible region, corresponding to the image of the beam blocker, at the center of the field of excitation [12]. Illuminating the LC-SLM with a diverging or converging beam and compensating the beam divergence with a Fresnel lens addressed to the LC-SLM permits to axially displace the zero order from the holographic pattern $P_{\text {out }}[13,14]$, therefore moving the dark region of the beam blocker away from the sample plane. However, this still reduces the accessible three-dimensional field of excitation. Alternatively, an elegant solution consists in adding a destructively interfering spot to the phase hologram design [15, 16] but this solution is not versatile since the phase and the amplitude of the required spot depend on the addressed phase pattern.

In this paper, we take advantage of the nonlinear dependence of the two-photon effect $[8,2]$ on the excitation intensity, and propose to reduce the zero-order contribution by intentionally 
introducing aberrations in the excitation beam that spread the zero-order spot over a larger area. Aberrations correction by the SLM then restores the original pattern, while leaving the zero-order component aberrated. We implement this approach by introducing astigmatism in a two-photon computer generated holography $(\mathrm{CGH})$ setup and demonstrate that the zero-order intensity at the sample plane can be reduced by 4 orders of magnitude without the need of a beam blocker or change in the beam divergence. We also demonstrate that introducing astigmatism in CGH systems using temporal focusing permits a 6 orders of magnitude decrease of the zero-order component.

Astigmatism is introduced here by inserting one or two cylindrical lenses, CLs, in the optical path of a holographic system illustrated in Figure 1. A liquid crystal SLM (LCOS-SLM X10468-02, Hamamatsu Photonics) is illuminated by a femtosecond Ti:Sapphire laser (MaiTai Deep-See, Spectra-Physics; $\lambda=800 \mathrm{~nm}$ ) and imaged at the back focal plane of a first $60 \times$, 0.9 NA objective (Olympus LUMPLFLN 60XW) through a $\times 1 / 2$ telescope composed by two lenses L1 $\left(f_{1}=1000 \mathrm{~mm}\right)$ and L2 $\left(f_{2}=500 \mathrm{~mm}\right)$. Holographic phase patterns, $\varphi_{\text {mask }}$, are computed using an iterative Fourier-transform algorithm, imposing uniform illumination in the Fourier plane and generating two-photon (2P) holographic light patterns in the image plane. A thin spin-coated layer of Poly-Methyl MethAcrylate (PMMA) doped with rhodamine-6G is placed at the sample plane. Fluorescence emitted by the rhodamine layer is collected by a second objective (Olympus UPlanSApo 60XW, NA 1.2) placed below the sample for imaging on a CCD camera. Axial distribution of the excitation beam is probed by axial scanning of the upper objective while keeping the lower one focused on the rhodamine layer. Combination of $\mathrm{CGH}$ and temporal focusing is achieved by replacing the mirror $\mathrm{M}$ in Figure 1 with a reflective ruled diffraction grating of $300 \mathrm{l} / \mathrm{mm}$ (Richardson Gratings, Newport). Astigmatism is introduced by placing CLs (of focal length $f_{c y l}$ ) either in front of the SLM (configuration A) or right before the first objective (configuration B). The aberrating phase, $\varphi_{a b b}$, introduced by CLs is compensated by adding the conjugated cylindrical Fresnel phase, $-\varphi_{a b b}$, to the holographic phase pattern, $\varphi_{\text {mask }}$. At the sample the aberrated zero order gives rise to two orthogonal lines separated by a distance $\Delta z=n\left(f_{o b j}^{2} / f_{e q}\right)$ along the axial direction, where $f_{o b j}$ is the focal length of the objective, $n$ the immersion refractive index and where 
$f_{e q}=\left(f_{2} / f_{1}\right)^{2} f_{c y l} / 2$ or $f_{e q}=f_{c y l}$, for configuration $\mathrm{A}$ or $\mathrm{B}$, respectively. The line length is then $L \simeq 2 \Delta z N A / n$, where $N A$ is the numerical aperture of the first objective.

In $2 \mathrm{P}$ excitation, the fluorescence signal scales squarely with the excitation intensity. For a given amount of incident power in the zero order, the $2 \mathrm{P}$-generated signal is therefore proportional to $1 / S^{2}$, where $S$ is the surface over which the power is spread. A CL in the optical path will therefore decrease the zero-order signal at the sample plane by $\mathrm{L}^{2} \propto 1 / \mathrm{f}_{\mathrm{cyl}}{ }^{2}$ and the zero-order signal integrated in the focal plane by $\mathrm{L} \propto 1 / \mathrm{f}_{\mathrm{cyl}}$.

An example of a 2P fluorescence image generated by a CGH pattern representing the Eiffel tower is shown in Figure 2a (corresponding phase hologram, $\varphi_{\text {mask }}$, in Figure 2d). In this figure, although the amount of light in the zero order is only a small percentage of the one diffracted into the rest of the image, the fluorescence intensity induced by the zero order is higher than the one generated within the holographic pattern (red lines in the graph of Figure 2c). This is mainly due to the large surface over which the diffracted light is spread relatively to the zero order. The addition of a CL of focal length $f_{c y l}=1 m$ in front of the SLM and a compensating cylindrical Fresnel phase, $-\varphi_{a b b}$, (of focal length $f=-f_{c y l} / 2=-500 \mathrm{~mm}$; Figure 2e) to the hologram $\varphi_{\text {mask }}$ (Figure 2d), leaves the holographic pattern practically unaffected

(Figure $\mathbf{2 b}$ and $\mathbf{2 c}$ blue lines), while generating a strongly aberrated zero-order component dispersed in a broad area. The corrected phase mask, $\varphi_{\text {corr }}$, is shown in Figure $2 \mathbf{f}$.

In order to precisely characterize the decrease in fluorescence caused by astigmatism, we switched off the SLM to send all the light in the zero order. Then we measured for different CLs the axial distribution of the zero order by moving the excitation objective axially in steps of $1 \mu \mathrm{m}$, and acquiring through the detection objective the fluorescence generated in the rhodamine layer. The intensity integrated in each plane is plotted in Figure $\mathbf{3}$ as a function of the axial position, for five different values of $f_{e q}$. Two peaks separated by $\Delta \mathrm{z} \simeq \mathrm{nL} / 2 N A$, corresponding to the planes where the two orthogonal lines are focused, appear clearly. As expected, the magnitude of the peaks is proportional to 1/L (inset of Figure 3). The fluorescence intensity measured by taking the peak intensity at the center of each line decreases proportionally to $1 / \mathrm{L}^{2}$. In the case of $\mathrm{L} \approx 130 \mu \mathrm{m}$ we observed a decrease by two orders of magnitude (from $\sim 200$ Counts $/ \mathrm{mW}^{2} / \mathrm{ms}$ to $\sim 2$ Counts $/ \mathrm{mW}^{2} / \mathrm{ms}$ ) in the integrated fluorescence compared to the non-aberrated spot, corresponding to a four orders of magnitude decrease in the fluorescence intensity (Figure 3 and inset). 
When introducing aberrations with a single cylindrical lens, one of the two focused lines still lies in the focal plane of the objective. Further reduction of the zero-order component in the focal plane can be achieved by adding a second cross-oriented cylindrical lens with opposite vergence. This configuration generates two lines at two planes, $P L_{1}$ and $P L_{2}$, on either side of the objective focal plane. Then, the zero-order spot is enlarged at the focal plane along the two transverse directions, as expected, further reducing $2 \mathrm{P}$ excitation by a factor of 100 . An example of the effect of this configuration is illustrated in Figure 4 (dashed line) where two CLs of $f_{c y l, 1}=1 \mathrm{~m}$ and $f_{c y l, 2}=-1 \mathrm{~m}$ are placed in contact, in front of the SLM (configuration A). The zero order then focuses two lines at $\sim 96 \mu \mathrm{m}$ on either side of the focal plane.

The remaining excitation by out of focus lines is further reduced in CGH systems confining 2P excitation in the focal plane thanks to temporal focusing $[17,18,19]$. In this case, mirror $\mathrm{M}$ in Figure 1, is replaced by a blazed grating which splits the spectral components of the femtosecond pulse in the back focal plane of the objective lens. The induced pulse broadening out of the focal plane, in particular at $P L_{1}$ and $P L_{2}$, permits reducing the maximum fluorescence signal to 0.1 Counts $/ \mathrm{mW}^{2} / \mathrm{ms}$ (Figure 4; solid line), suggesting a $2.10^{3}$ decrease in integrated fluorescence intensity and a $4.10^{6}$ decrease in the fluorescence intensity. In this example, the two CLs of $f_{c y l, 1}=1 \mathrm{~m}$ and $f_{c y l, 2}=-1 \mathrm{~m}$ are oriented at $+45^{\circ}$ and $-45^{\circ}$ relatively to the grating lines, which provides optimal suppression of the out-of-focus fluorescence.

The presented results demonstrate the efficiency of the proposed approach to suppress excitation by the zero-order component produced by a LC-SLM. Previous techniques consisted mainly in spatially separating the zero from the first diffraction order, which corresponds to the target spot, and physically blocking the zero order. Here, zero-order suppression is achieved by spreading this component on a large area by the use of an aberrating element in combination with non-linear two-photon excitation. Compensation of the induced aberration with a suitable phase profile at the SLM then permits to fully restore the quality of the hologram. For choosing the aberrating element a compromise between the desired decrease in the zero-order excitation and the ability of the SLM to correct the introduced aberrations needs to be found because the SLM can only reproduce a discretized approximation of the exact phase profile that correct the aberrations. Typical spatial scale of phase variations must not be smaller than the pixel size, $a$, of the SLM and the phase difference between adjacent pixels must preferably be less than $\pi$. For aberrations exceeding these limits, correction by the SLM is not optimal and is done at the expense of a significant loss in diffraction efficiency especially for patterns projected at the 
center of the field of excitation. For a cylindrical Fresnel phase, the minimum focal length that can be addressed to the SLM is $f_{C}=N a^{2} / \lambda$ [12], where $N$ is the number of pixels of the SLM that fill the objective back aperture. In the present optical system, this value is $f_{c}=270 \mathrm{~mm}$ allowing the use of a minimum focal length, $f_{c y l}$, of $540 \mathrm{~mm}$ (due to the double pass) in configuration A or $135 \mathrm{~mm}$ in configuration $\mathrm{B}$. When using a $\mathrm{CL}$ of $f_{c y l}=1 \mathrm{~m}$ in this same configuration (or a $f_{c y l}=125 \mathrm{~mm}$ in configuration $\mathrm{B}$ ), which corresponds to $\mathrm{L} \approx 130 \mu \mathrm{m}$, we measured for a spot placed at the center of the field of view a decrease in diffraction efficiency of $\sim 12 \%$. Finally, we point out that the proposed technique is also efficient for single photon processes exhibiting a threshold level (optical tweezers, photo-polymerization, cellular photo-activation), since the intensity of the zero order may then be diminished below the excitation level.

In conclusion, we have demonstrated that efficient suppression of the zero-order component in an optical system for CGH can be achieved by introducing aberrations in the optical design to redistribute the zero-order in a larger area. In particular, the use of one or two crossed cylindrical lenses as aberrating elements permits reducing by 4 to 6 orders of magnitude the fluorescence generated by the zero-order component at the focal plane. Optical quality of the holographic system is fully restored by achieving aberration correction with the SLM. Finally, we have shown that the configuration with two crossed CLs combined with TF permits an almost total suppression of the background signal in the sample volume.

Our approach is characterized here with the simple case of astigmatism. Comparatively, more elaborated aberrations could enlarge the zero order along the two transverse dimensions then further improving the decrease in fluorescence intensity.

Figure 1: (a) Principle of a CGH microscope. An SLM is conjugated to the back focal plane of an objective lens (OBJ1) with a telescope (L1, $f=1 \mathrm{~m}, \mathrm{~L} 2, f=500 \mathrm{~mm}$ ). The light beam $(\lambda=800 \mathrm{~nm})$ arising from a femtosecond Ti:Sapphire laser induces two-photon fluorescence in a rhodamine layer. An image of the sample is made on a CCD camera through a second opposite objective (OBJ2) and a tube lens (L3). Astigmatism is introduced in the system by adding cylindrical lenses (CL) either in front of the SLM (configuration A) or near the back aperture of the first objective (configuration B). For temporal focusing, the mirror M, which lies in the object focal plane of L2 is replaced by a blazed grating. 
Figure 2: Comparison between a computer-generated holographic image of the Eiffel tower without (a) and with (b) a single $1 \mathrm{~m}$ cylindrical lens aberrating the zero order in the optical path. (c) 2P Normalized Fluorescence Intensity (NFI) profiles along the lines drawn in (a) (red) and (b) (blue). Dotted lines represent the signal of solid lines multiplied by 10 for better view. (d) Phase mask, $\varphi_{\text {mask}}$, reproducing the image of the Eiffel tower at the focal plane of the objective. (e) Conjugated cylindrical Fresnel lens hologram, $-\varphi_{a b b}$, added to the one of (d) for aberration compensation. (f) Final corrected phase mask, $\varphi_{\text {corr }}$, addressed to the SLM.

Figure 3: Integrated two-photon Fluorescence Intensity (IFI) of the zero order as a function of the distance from the focal plane, for five different cylindrical lenses. The distance $\Delta z$ increases with the optical power of the cylindrical lens while induced fluorescence decreases. Inset shows the evolution of the fluorescence (FI) and the integrated fluorescence intensity (IFI) in the focal plane ( $\mathrm{z}=0$ ) as a function of $\Delta z$, in logarithmic scale. A slope of -1.0 is obtained for the IFI and -2.0 for the FI, in agreement with theory. Values of $\Delta z$ ranging from $12 \mu m$ to $37 \mu m$ have been obtained by placing CLs of different focal lengths in configuration B shown in Figure $1 . \Delta z=96 \mu \mathrm{m}$ has been obtained by placing a $1 \mathrm{~m}$ focal length in configuration A.

Figure 4: Integrated two-photon Fluorescence Intensity (IFI) of the zero order as a function of the distance from the focal plane. The zero order is here aberrated with two cross-oriented CLs (dashed red) in configuration A, with focal length $+1 m$ and $-1 m$. Combination with temporal focusing (solid blue) suppresses fluorescence of the two lines as the excitation pulse is chirped in these planes.

\section{Acknowledgments}

We thank V. de Sars for adapting our custom made software 'Wavefront Designer IV' to the needs of the experiments and B.C. Forget for critical reading of the paper. VE acknowledges financial support by the 'Fondation pour la Recherche Médicale' (FRM Équipe) and the French National Research Agency (grants ANR-12-BSV5-0011-01, Neurholog and France-BioImaging Infrastructure ANR-10-INSB-04, Investments for the future). OH acknowledges the program Nanotechnologies France-Israël for financial support and the doctoral school 'Frontiers in Life Sciences'. 


\section{References}

[1] E. Dufresne, G. Spalding, M. Dearing, S. Sheets, and D. Grier, "Computer-generated holographic optical tweezer arrays," review of scientific instruments 72, 1810-1816 (2001).

[2] L. Kelemen, P. Ormos, and G. Vizsnyiczai, "Two-photon polymerization with optimized spatial light modulator," Journal of the European Optical Society - Rapid publications 6 (2011).

[3] P. Wang and R. Menon, "Three-dimensional lithography via digital holography," in "Frontiers in Optics 2012/Laser Science XXVIII," (Optical Society of America, 2012), p. FTu3A.4.

[4] C. Lutz, T. S. Otis, V. DeSars, S. Charpak, D. A. DiGregorio, and V. Emiliani, "Holographic photolysis of caged neurotransmitters," nature methods 5, 821-827 (2008).

[5] B. Xu, J. Gunn, J. Dela Cruz, V. Lozovoy, and M. Dantus, "Quantitative investigation of the multiphoton intrapulse interference phase scan method for simultaneous phase measurement and compensation of femtosecond laser pulses," journal of the optical society of america b-optical physics $\mathbf{2 3}, 750-759$ (2006).

[6] C.-L. Hsieh, Y. Pu, R. Grange, and D. Psaltis, "Second harmonic generation from nanocrystals under linearly and circularly polarized excitations," Opt. Express 18, 1191711932 (2010).

[7] I. M. Vellekoop, M. Cui, and C. Yang, "Digital optical phase conjugation of fluorescence in turbid tissue," applied physics letters 101 (2012).

[8] D. Debarre, E. J. Botcherby, T. Watanabe, S. Srinivas, M. J. Booth, and T. Wilson, "Image-based adaptive optics for two-photon microscopy," optics letters 34, 2495-2497 (2009).

[9] S. M. Popoff, G. Lerosey, R. Carminati, M. Fink, A. C. Boccara, and S. Gigan, "Measuring the Transmission Matrix in Optics: An Approach to the Study and Control of Light Propagation in Disordered Media,” PHYSICAL REVIEW LETTERS 104 (2010).

[10]E. Ronzitti, M. Guillon, V. de Sars, and V. Emiliani, "LCoS nematic SLM characterization and modeling for diffraction efficiency optimization, zero and ghost orders suppression," Opt. Express 20, 17843-17855 (2012).

[11] R. W. Gerchberg and W. O. Saxton, "A practical algorithm for the determination of the phase from image and diffraction plane pictures," Optik 35, 237-246 (1972).

[12] L. Golan, I. Reutsky, N. Farah, and S. Shoham, "Design and characteristics of holographic neural photo-stimulation systems," journal of neural engineering 6 (2009). 
[13] M. Zahid, M. Velez-Fort, E. Papagiakoumou, C. Ventalon, M. C. Angulo, and V. Emiliani, "Holographic Photolysis for Multiple Cell Stimulation in Mouse Hippocampal Slices," PLOS ONE 5 (2010).

[14] M. Polin, K. Ladavac, S. Lee, Y. Roichman, and D. Grier, "Optimized holographic optical traps," Opt. Express 13, 5831-5845 (2005).

[15] D. Palima and V. R. Daria, "Holographic projection of arbitrary light patterns with a suppressed zero-order beam," applied optics 46, 4197-4201 (2007).

[16] A. Jesacher and M. J. Booth, "Parallel direct laser writing in three dimensions with spatially dependent aberration correction," Opt. Express 18, 21090-21099 (2010).

[17] D. Oron, E. Tal, and Y. Silberberg, "Scanningless depth-resolved microscopy," Opt. Express 13, 1468-1476 (2005).

[18]E. Papagiakoumou, V. de Sars, D. Oron, and V. Emiliani, "Patterned two-photon illumination by spatiotemporal shaping of ultrashort pulses," Opt. Express 16, 22039-22047 (2008).

[19] A. Begue, E. Papagiakoumou, B. Leshem, R. Conti, L. Enke, D. Oron, and V. Emiliani, "Two-photon excitation in scattering media by spatiotemporally shaped beams and their application in optogenetic stimulation," Biomed. Opt. Express 4, 2869-2879 (2013). 
